\title{
Advanced Non-Destructive Testing in Steels
}

\author{
Evangelos Hristoforou
}

Laboratory of Electronic Sensors, National Technical University of Athens (NTUA), Zografou Campus, 15780 Athens, Greece; hristoforou@ece.ntua.gr

Received: 21 June 2018; Accepted: 26 June 2018; Published: 28 June 2018

\section{Introduction}

The quality of steel and its corresponding products depends on the distribution and level of stress on the steel's volume and surface, since the stress gradient is responsible for steel cracking generation and failure [1,2]. The industrially existing technology for stress monitoring concerns either surface stress distribution monitoring instruments, with unacceptably high uncertainty, or point surface stress sensors. Laboratory tests, apart from the classic diffraction techniques like X-ray and neutron diffraction, mainly include magnetic, acoustic, and electromagnetic techniques.

The steel market today involves the following:

- $\quad$ Steel producers like steel coil and rod producers, as well as steel manufacturers (pipelines, metal forming, etc.)

- Transport, with an emphasis on shipyards and shipping companies, as well as the railway and the automobile industry

- Power plants, including nuclear stations as well as the oil and gas industry (recovery, refineries, etc.)

- The construction sector, including metallic buildings, bridges, water pipelines, etc.

- Inspection bodies, including classification societies, testing laboratories, regulatory bodies, etc.

The steel market occupies more than $5 \%$ of the global GDP, a percentage that is increasing due to advances in the recycling technology of scrap steel. Steel is produced in steel coils and rods, and is manufactured into products in shipyards, railway and train factories, pipelines, the automotive and train industry, etc. All of these industrial sectors need quality inspection and monitoring techniques.

Therefore, non-destructive testing (NDT) methods that provide stress tensor distribution monitoring on the surface and within the bulk of steel, therefore leading to steel health monitoring, are under investigation in order to meet the needs of the steel industry. These methods could also be embedded in a feedback system for the stress rehabilitation (annihilation or strengthening) process in steel production and manufacturing, as well as the monitoring of installed steel structures (end-user applications).

\section{Contributions}

In the current special issue, the two governing NDT methods, namely magnetic and acoustic ones offering several possibilities for stress and steel health monitoring, are examined. Apart from those, electric and electromagnetic methods, which have also contributed towards such health monitoring, are presented as well.

Concerning the magnetic methods, the review paper provides an excellent description of the dependence of the magnetization loop and Barkhausen noise on the stress state of ferromagnetic steels. According to these measurements, several parameters of the magnetization loop, such as coercivity, losses, permeability, etc. can be used to correlate the stress state with magnetic properties. However, the 
non-monotonic behavior of these parameters may lead to uncertainties during monitoring. Barkhausen noise $(\mathrm{BHN})$ results provide very sensitive information, illustrating that the method can be used as a precise laboratory tool for steel investigation. Apart from that, the classic and fundamental scaling laws developed by the late Professor Takahashi, which refer to the scaling law dependence of magnetic characteristics on the dislocation density of magnetic steels, offer fundamental information about the role of the dislocation density distribution and anisotropic orientation on the magnetic properties' dependence on stress. The third paper refers to the metal magnetic method (MMM), according to which the stress state of the steel, both on the surface and within the bulk, opposes or assists the intrinsic magnetization of the steel, and can be detected as a (mainly) surface magnetic field, with no need for any type of electromagnetic excitation.

One of the articles referenced concerning acoustic methods refers to the dependence of acoustic parameters on the stress state of austenitic steels. For this type of steel, which does not possess magnetic properties but can provide acoustic information, the authors managed to correlate the acoustic parameters with the microstructure of the austenitic steel. Apart from that, the second paper on acoustics refers to guided acoustic waves. This method of combining phased arrays can provide a detailed topology of microstructural changes in metals and steels, thus permitting steel health monitoring; the proper selection of excitation frequencies and amplitudes can offer monitoring of stress distribution. The third paper on acoustics refers to acoustic emission detection and spatial monitoring, according to which even the generation of dislocation can be detected. This technique, initiated in the early 1960s, is still a stress initiation technology in metals, and particularly in steels. Talking about acoustic methods, it is worth mentioning the non-linear acoustic principles postulating that the localized stresses may be determined by monitoring the non-linear parameters of acoustic waves and evaluating their contribution to the non-linear behavior of the sound velocity in the material.

Finally, electric and electromagnetic methods are presented. The first refers to current-voltage dependence on the corrosion of steels, using the electrolytic cell principle. The method can provide corrosion monitoring, which is certainly interesting for several applications. The second one offers the possibility of using neural networks to process data from eddy current sensors, thus offering the possibility of correlating data from different surface areas of the examined steel.

\section{Conclusions}

Some of the current advances on NDT stress-monitoring distribution in steels have been presented in this special issue. A number of these methods and instruments, like the magnetization loop or MMM measurements, guided waves, and classic acoustic transducers, as well as eddy currents, can be used in industrial environments, providing correlation with localized stress states in an industrial environment. The rest of the NDT methods presented, like BHN, the scaling law, and I-V electrolytic monitoring can be used in laboratory applications.

However, new NDT and corresponding methods must be developed with the ability to accomplish fast stress distribution monitoring, which will allow for their operation as feedback systems for the steel rehabilitation process.

Conflicts of Interest: The author declares no conflict of interest. 


\section{References}

1. Dobmann, G.; Meyendorf, N.; Schneider, E. Nondestructive characterization of materials a growing demand for describing damage and service-life-relevant aging processes in plant components. Nucl. Eng. Des. 1997, 171, 95-112. [CrossRef]

2. Hristoforou, E.; Vourna, P.; Ktena, A.; Svec, P. On the Universality of the Dependence of Magnetic Parameters on Residual Stresses in Steels. IEEE Trans. Magn. 2016, 52, 7362189. [CrossRef]

(c) (2)

(c) 2018 by the author. Licensee MDPI, Basel, Switzerland. This article is an open access article distributed under the terms and conditions of the Creative Commons Attribution

(CC BY) license (http://creativecommons.org/licenses/by/4.0/). 